\title{
A COGNITIVE APPROACH TO TEACHING A GRADUATE-LEVEL GEOBIA COURSE
}

\author{
Raechel A. Bianchetti ${ }^{\mathrm{a}}$ \\ aDepartment of Geography, Michigan State University, East Lansing, MI, USA - bnketti@ msu.edu
}

Commission VI, WG VI/2

KEY WORDS: GEOBIA, Education, Cognition, Open Source

\begin{abstract}
:
Remote sensing image analysis training occurs both in the classroom and the research lab. Education in the classroom for traditional pixel-based image analysis has been standardized across college curriculums. However, with the increasing interest in Geographic Object-Based Image Analysis (GEOBIA), there is a need to develop classroom instruction for this method of image analysis. While traditional remote sensing courses emphasize the expansion of skills and knowledge related to the use of computer-based analysis, GEOBIA courses should examine the cognitive factors underlying visual interpretation. This current paper provides an initial analysis of the development, implementation, and outcomes of a GEOBIA course that considers not only the computational methods of GEOBIA, but also the cognitive factors of expertise, that such software attempts to replicate. Finally, a reflection on the first instantiation of this course is presented, in addition to plans for development of an open-source repository for course materials.
\end{abstract}

\section{INTRODUCTION}

Geographic Object Based Image Analysis (GEOBIA) is becoming accessible to a greater number of remote sensing practitioners (Hay and Castilla 2006). GEOBIA is finding favor over per pixel methods in some use cases for a variety of reasons, one being its close relationship to expert reasoning abilities (Blaschke et al. 2014). Given this relationship between human reasoning and computational modelling, it would follow that practitioners of GEOBIA would benefit from a deep understanding of the cognitive processes that facilitate visual interpretation. Knowledge of these cognitive processes should improve the ability of practitioners developing models of them. However, as remote sensing has continued to depend on automated analysis, there has been shift in focus of remote sensing courses towards computational methods and away from visual interpretation (Gardin et al. 2011, Sader and Vermillion 2000, Sader and Mueller 2012).

While literature on GEOBIA champions the strengths of human reasoning (contextual development, object recognition, etc.) in developing insights from remote sensing imagery. We hypothesize that a shift away from developing visual interpretation skills and knowledge in the classroom impedes the practitioner from fully exploiting the strengths of GEOBIA. The goal of this current paper is to describe an approach to designing a GEOBIA course that integrates cognitive science concepts from air photo interpretation and geographic visualization with core competencies from previous initiatives in curricular design. The remainder of the paper outlines the previous research concerning remote sensing curriculum. This is then followed by an examination of core competencies in spatial thinking that are pertinent to GEOBIA. A description of the course content is also provided. Finally, a discussion of the challenges and triumphs from the first offering of the course is provided along with improvements that are being made for the next offering.

\section{REMOTE SENSING EDUCATION}

Remote sensing is not a purely geographic education initiative, trends in remote sensing education are found across a number of disciplines. A review of findings across the forestry and geography disciplines gives an extended look at the thematic trends developing in the curriculum. A major trend that has been identified is the decrease in instruction on visual image interpretation, in favor of training in GIS and computer-based methods of analysis (Sader and Mueller 2012).

According to Jensen (2001), an ideal student of remote sensing would begin image analysis training in latter stages of their primary education; however, with limited accessibility to raw remote sensing data at such a young age most remote sensing education takes place in secondary education. With a lack of a standardized remote sensing curriculum, students are exposed to a wide range of training, suggesting a need for curriculum standardization to meet the demands of the workforce (Jensen 2001).

Reviews of remote sensing education within the forestry discipline have found a decrease in air photo interpretation and photogrammetry training (Sader and Mueller 2012, Sader and Vermillion 2000). Despite this decrease, forestry employers still desire practitioners with strong photo interpretation skills, ranking above GIS and computer programming (Brown and Lassoie 1998). In their 1998 survey, (Sader and Vermillion 2000) found that the majority of forestry remote sensing courses required Aerial Photography and Image Interpretation (Paine 1981), a text emphasizing human interpretation. However, this was not the case during their next implementation (2011) of the remote sensing course survey. Instead, a much more computationally oriented book was used, Remote sensing and image interpretation (Lillesand, Kiefer and Chipman 2004). Sader and Mueller suggest that this shift may be due to instructor unfamiliarity of air photo interpretation techniques, or increasing reliance on computer-based image analysis (Sader and Mueller 2012). 
In their 2011 survey, (Sader and Mueller 2012) found that about $1 / 3$ of remote sensing courses did not teach air photo interpretation. The study also revealed that only about $4.8 \%$ of respondents taught the use of eCognition, a popular GEOBIA tool. This suggests two gaps in remote sensing education, both GEOBIA and visual interpretation methods.

Trends in remote sensing course content have shifted over the past twenty years. Photo interpretation and texts focusing on photo interpretation have received less focus in current remote sensing courses over time. This change in focus is unfortunate given the emphasis of GEOBIA literature on the importance of human interpretation (Blacschke, Lang and Hay 2008, Blaschke et al. 2014)

\section{CIRICULUAR STANDARDIZATION}

Development of consistent, comprehensive, and appropriate curriculum for remote sensing education is paramount in order to develop a workforce consisting of competent members. A number of initiatives for the standardization of key competencies within GIScience, and more specifically remote sensing provide frameworks for designing such courses. The following section describes several of these initiatives.

\subsection{GIScience Curriculum}

A number of initiatives for defining core curricular elements in geospatial sciences have been developed. The majority of these initiatives are aimed at the broader geospatial science community. For example, the OSGeo group's Education and Curriculum committee has organized geoforall.org, a collection of materials for education across primary and secondary education (Sui 2014). The NCGIA Core Curriculum in GIScience, a collaboration between the University of California Santa Barbara, SUNY- Buffalo, and the University of Maine, has led to the development of a number of initiatives to improve undergraduate education and collect teaching resources for educators (Estes et al. 1993). Developed by the UCGIS Education Committee, the Geographic Information Science and Technology Body of Knowledge is a compilation of over 300 GIScience topics and 1600 learning objectives for GIScience educators (DiBiase et al. 2007). The Body of Knowledge is likely the most comprehensive guidelines for GIScience education ( $\mathrm{du}$ Plessis and Van Niekerk 2014, Reinhardt 2013).

\subsection{Remote Sensing Curriculum}

Complementing the NCGIA Core Curriculum in GIScience, the Remote Sensing Core Curriculum was produced by the International Center for Remote Sensing Education (ICRSEdu) was developed to meet the need for a national-level core remote sensing curriculum based on guidelines from the American Society of Photogrammetry and Remote Sensing (ASPRS), National Center for Geographic Information and Analysis (NCGIA), the National Aeronautics and Space Agency (NASA), and the Earth Observation Satellite Company (EOSAT) (Estes et al. 1993). The curriculum has been developed by a number of the experts in the remote sensing field from both academe and industry (Foresman and Serpi 1999). The results of this initiative are available through two different web portals, but the most recent contributions seem to be outdated, the suggestions are not mandated, and the modules are incomplete (Jensen 2001). Other attempts to develop comprehensive remote sensing curricular materials through contributions by experts in the field include the Institute for
Advanced Education in Geospatial Sciences. Similar to other initiatives, the goal was to provide courses created by content experts to as many colleges and universities as possible (Lawhead and Johnson 2003).

These initiatives are designed to convey the knowledge and technical skills necessary to be successful in the field of geospatial science. Assessment of remote sensing programs, by Sader (2012) found that a third of respondents do not cover air photo interpretation in their courses. Their results also suggested a decrease in the amount of class time dedicated to photographic interpretation and non-photographic interpretation compared to an earlier 1988 study. From these results, Sader suggests that remote sensing courses should emphasize aerial photo interpretation.

\subsection{Competencies for GEOBIA}

An initial survey of curriculum design initiatives has suggested a lack of attention to the role of GEOBIA in the curriculum. The Sader (2012) found that Trimble's eCognition was taught in $4.8 \%$ of courses. Beyond this, not much information is available regarding the core GEOBIA curriculum. This is unfortunate given the strong connection between GEOBIA and visual interpretation, as it may be possible to use GEOBIA to increase student skills in visual interpretation.

Previous surveys of remote sensing curriculum have shown a shift in emphasis from photo interpretation to computational methods of information extraction. Additionally, training of practitioners is made difficult by a lack of standardization. Finally, with increasing availability of GEOBIA software, there is a need to develop courses to train novices in this method. The remainder of this paper describe a systematic approach to designing a GEOBIA course that re-emphasizes the role of human-based interpretation while training graduate students in GEOBIA

\section{GEOBIA CIRRICULUM}

A survey of the GIS\&T Body of Knowledge (David DiBiase et al 2006) was completed and a set of core competencies necessary for analysts implementing GEOBIA was identified. It was decided that two core components would be used to structure the course. The first component is that of spatial thinking, human cognitive methods of understanding imagery. Second, computerassisted image analysis, the use of computer technology to perform image analysis. In the case of the second area, GEOBIA was chosen as the main analytical paradigm.

\subsection{GIS\&T Body of Knowledge}

The knowledge areas, as prescribed by the GIS\&T BoK, suggest groups of related skills, knowledge, and applications. Within each of the knowledge areas is a set of units, comprised of topics related to the overarching knowledge area. The units are competencies that a graduate should have. Each unit then has set of topics. These topics consist of a number of educational outcomes, called objectives. The objectives can be used to structure course materials and formalize course assessments (David DiBiase et al. 2006)

This Body of Knowledge was used to determine what skills and training a graduate student successfully completing a GEOBIA course would require. Eight of the Knowledge Areas were 
determined to be important for the course design. Within these 8 knowledge areas, 27 units were identified. At the end of the survey, over 30 educational objectives were identified. A sample of some of the objectives identified follows:

- Compare and contrast different measures of distance

- Compare and contrast different shape indices

- Collaborate effectively with colleagues from different social backgrounds

- Describe the perceptual processes that aid in cognitive objectification

- Create appropriate membership functions to model vague phenomena

The objectives identified through the survey were used to guide the development of course material. For example, the objective identified above 'Compare and contrast different shape indices' was linked to a tutorial on the use of the geometric measurements available in eCognition. In another example, the objective of 'Collaborate effectively with colleagues from different social backgrounds' was assessed through a semester- long group project.

\section{COURSE IMPLEMENTATION}

In the fall semester of 2015 a graduate course on GEOBIA was offered to students across the Michigan State University campus. No pre-requisites were required for the course during this initial offering due to the fact that it was offered under a pre-existing course number. A description of the course is provided below.

\subsection{Course Topics}

Five major units were presented in the course; remote sensing primer, visual perception, image segmentation, knowledge representation, and classification. Following the core content of the course (12 weeks in length), the remaining three weeks were spent on the students' final projects. Each of the computational units was paired with a corresponding cognitive unit. A description of the content within each of these topics follow.

5.1. Remote Sensing Primer The first two weeks of the course provided a primer on basic topics of remote sensing. These topics correspond to Remote Sensing Core Curriculum Volume 2, Overview of Remote Sensing of Environment. Students were provided with a brief background on the development of remote sensing, with an emphasis on interpretation developments during World War II. Next, the students were led through topics on the electromagnetic spectrum and resolutions. While these topics should be covered directly in undergraduate education, it was necessary given the lack of pre-requisites for the current instantiation. The final section of this unit is the introduction of the two major analysis paradigms: pixel-based and object-based image analyses.

In addition to course lectures and readings on these topics, students complete several tutorials using eCognition (2014) related to the basic eCognition environment and setting up a workspace for analysis. Topics included how to open imagery, update metadata, and switch between different interface presets.
5.12 Visual Perception The second two-week section of the course addresses human visual perception. This section addresses both cognitive GIScience research on image interpretation [for example (Lloyd 2002)] as well as early training materials used to teach photo interpretation [for example, (Stone 1964)]. The physiological process of visual perception is explained as well as different theories visual interpretation, physiology of the eye, and visual illusion. For example, the concept of relief inversion is covered and the psychological explanation of the phenomena is presented. The goal of reading these materials is for students to understand the psychological factors that underlie their interpretation abilities.

In addition to the course lectures and readings on these topics, students complete several visual perception exercises. Examples of potentially difficult interpretation situations are presented and discussed.

5.13 Image Segmentation The next, three-week section of the course addresses image segmentation methods. It is at this point that a multi-disciplinary perspective is best taken. It offers a chance to tie automated analysis to topics on visual search. A large body of research and descriptions of image segmentation algorithms exist outside of remote sensing. Texts from both remote sensing and medical digital image processing were used to provide cursory information on segmentation. For example, the differences between edge and area based segmentation routines [for example, (Dougherty 2009, Navulur 2006a)]. Additional readings from peer reviewed journals were also provided.

This section of the course the first graded lab assignment was given. Students carried out the segmentation of two NAIP images, one of a rural area and another in an urban setting. They were asked to use each of the image segmentation algorithms available in eCognition, and vary several parameters for each in order to visualize the effects of the parameters on segmentation outcomes. Students were asked to write a brief comparison of the algorithm outcomes to how they visually perceive objects in the images. This activity links the previous section on visual interpretation to computation.

5.14 Knowledge Representation The next two weeks addressed the topic of knowledge representation. This unit included both internal representations of reality (conceptualization) and external descriptions (semantics) and models (ontologies) of geographic knowledge. Several key land cover/land use papers are used in conjunction with cognitive GIScience papers to present the concept of semantics and ontologies [for example (Ahlqvist 2004)]. While most geography students are familiar with land cover, and the use of papers on knowledge seems to help them transition to thinking about more abstract knowledge models.

No tutorials were provided to re-emphasize this topic area Though in the future, it would be beneficial to develop such activities. Instead, students were provided an initial lab on rulebased image classification. This exercise consisted of two parts. In the case of the classification exercise, students were first walked through the analysis of a pre-selected NAIP image, and in the second part the student was instructed to carry out a similar process on a new image of their choice. 
5.15 Classification The following three weeks addressed the classification methods used in GEOBIA. Rule-based classification was introduced first using (Navulur 2006b) and a number of recently published applied papers [For example, (Tong Yang, Liu and Gao 2015)]. Students were introduced to a number of statistical classifiers as well. Less focus was applied to machine learning algorithms, though that is a topic for further development as the course matures.

The final lab assignment assessed students' ability to think creatively to overcome challenges associated with urban environments, (such as the need for contextual information and inter-object heterogeneity). A NAIP image of Tempe, Arizona was provided and students were tasked with counting the number of residences within a one mile distance from a local city park.

\subsection{Student Assessment Methods}

The main methods of conveying information to students was inclass lectures and hands-on tutorials. The final assessment used for the course was comprised of Lab Activities - 50\%, Group Project $-15 \%$, Individual Project- 25\%, and Participation- $10 \%$. Participation for the course was judged by a student's active contribution during a number of in-class activities. Details for student assessments follow.

521 Tutorials These were designed to be completed in a short amount of time in class and did not have a grade associated with them. Students were encouraged to experiment with the software without fear of penalty. Topics covered in these tutorials included challenges associated with visual interpretation, organizing rulesets, image segmentation basics, and feature selection.

522 Lab Exercises Graded labs using Trimble's eCognition were assigned. These assignments typically were 2-3 weeks in duration. The final product for each of the labs included both the results of a classification (or segmentation in the case of the first lab) and a written response that tied the computational method back to the related cognitive topics. For example, in the case of the second lab, students compared the results of their final image classification to a map that they created using visual interpretation.

523 Projects Two final projects were assigned, a group project and an individual one. Two groups of students were formed based on topical interest. Each group was tasked with developing a research paper that would be submitted for publication or to a professional conference at the end of the semester. In the second project, students worked on small processing tasks that could be used to advance their own graduate research. While students were not expected to submit their individual projects for publication or conference presentation, it was also a feasible option for many.

For each of the final projects students took part in a number of activities with their fellow students to improve their research. For example, each student presented one paper from their literature review to the rest of the class and led a discussion of the topic. In another group exercise, students presented their initial hypotheses and planned workflow to their peers for assessment.

\section{OUTCOMES}

A graduate course in GEOBIA was designed and implemented during the fall semester of 2015. In designing the course two main objectives were identified. The first objective was to include a substantial portion of the course on air photo interpretation skills. This was seen as an important component since surveys have shown that traditional remote sensing curriculum has turned away from photo interpretation instruction (Sader and Mueller 2012), despite the influence of visual interpretation on designing GEOBIA analysis methods. The second objective was to implement strategies to integrate core knowledge components identified in previous research concerning remote sensing and GIS curriculum standardization. This early attention to core competency in the development of the course should improve the learning outcomes for course participants. The results of this initial course offering are described below. A formal assessment of outcomes was not performed, however, plans for a formal assessment of student learning outcomes is planned for a future offering.

\subsection{Positive Outcomes}

While there are number of positive outcomes for students in having a GEOBIA course available to them, here we highlight four of them. First, the availability of a course dedicated to the GEOBIA paradigm provides students with a unique opportunity to develop competency in a topic not traditionally a core component of introductory remote sensing courses. Second, in the absence of alternative courses on spatial thinking, this course serves as an introduction to a number of concepts that are not directly addressed in other courses within the department curriculum. Third, since the course did not require any prerequisite courses it was possible for students from programs across campus to come together in an inter- disciplinary, collaborative environment. Finally, the course has produced a variety of interesting papers that should be submitted for peer review publication.

\subsection{Necessary Improvements}

As with any initial course offering, there is much room for development and improvement. First and foremost, the development of appropriate resources for students is a continuous process, and one that is difficult to complete during the implementation of a course. New, more consistent, materials are currently being developed for the next implementation. These materials including tutorials, labs, lectures, and video tutorials will be made available using the open source tool, GitHub Second, a better connection between core competencies identified by the Body of Knowledge are necessary. While the BoK was used to inform the current design, the relation of these objectives to actual course content were not made explicit to students. In future material these core competencies will be identified throughout the course content and used to improve assessment strategies. Third, there is a noticeable absence of consistent training materials for GEOBIA education, and remote sensing training in general. The text by Navular is outdated, and some of the examples in the text are no longer functional with the latest edition of eCognition. A new text with focus on GEOBIA would make it easier to develop courses. A great deal of time was spent pulling together sources for this course. This is not only time consuming, but a very haphazard method for compiling and conveying knowledge. Fifth, the cost of the software is a major pitfall for implementing a GEOBIA course. Many universities simply do not have the funds available for purchasing and maintaining eCognition. Until a less expensive software is a viable option and has the training resources supporting it, this will be the biggest impediment. Finally, it is necessary to make a stronger case for 
the value of cognitive science topics in a remote sensing course. There is indeed an inherent value in including the topics in remote sensing courses that are shifting away from air photo interpretation; however, work should be done to tie these cognitive theories to GEOBIA practices. This is currently an active area of research for the author.

\section{CONCLUSIONS}

The initial offering of this course on GEOBIA to graduate students was a learning experience for both the students and the course instructor. While a formal assessment of the learning outcomes was not undertaken, the course evaluation for the course was 1.24 [1.0-highest, 5.0-lowest]. In the future, student learning outcomes will be assessed to evaluate the effectiveness of the curriculum. A second major component of these improvements will be opening up the course content for the rest of the remote sensing community.

\section{FUTURE WORK}

In talking with a number of members of the remote sensing community, it seems that initiatives are underway to update the BoK and the Remote Sensing Core Curriculum. Additionally, in speaking with other educators overseeing GEOBIA education, there are efforts being undertaken to develop standards for this specialty area as well.

\section{REFERENCES}

eCognition 9.0. Trimble Navigation, Sunnyvale, California. Blacschke, T., S. Lang \& G. H. Hay. (2008) Object-Based Image Analysis. In Lecture Notes in GeoInformation and Cartography. Hiedelberg, GR: Springer.

Blaschke, T., G. J. Hay, M. Kelly, S. Lang, P. Hofmann, E. Addink, R. Queiroz Feitosa, F. van der Meer, H. van der Werff \& F. van Coillie (2014) Geographic Object- Based Image AnalysisTowards a new paradigm. ISPRS Journal of Photogrammetry and Remote Sensing, 87, 180-191.

Brown, T. L. \& J. P. Lassoie (1998) Entry-Level Competency and Skill Requirements of Foresters: What Do Employers Want? Journal of Forestry, 96, 8-14.

David DiBiase, Michael DeMers, Ann Johnson, Karen Kemp, Ann Taylor Luck, Brandon Plewe \& E. Wentz. (2006) GIS and Technology Body of Knowledge. Washington, D.C.

DiBiase, D., M. DeMers, A. Johnson, K. Kemp, A. T. Luck, B. Plewe \& E. Wentz (2007) Introducing the first edition of geographic information science and technology body of knowledge. Cartography and Geographic Information Science, 34, 113-120.

Dougherty, G. (2009) Image Segmentation. In Digital image processing for medical applications. Cambridge University Press. du Plessis, H. \& A. Van Niekerk (2014) A new GISc framework and competency set for curricula development at South African universities. South African Journal of Geomatics, 3, 1-12.

Estes, J., J. Star, M. Goodchild, T. Cary, R. Eastman, N. Faust, T. Foresman, J. Jensen \& T. Shupin. (1993) The NCGIA Core Curriculum in Remote Sensing. 945- 948. American Society of Photogrammetry and Remote Sensing.

Foresman, T. \& T. Serpi (1999) Mandate for remote sensing education and the remote sensing core curriculum. Geocarto International, 14, 81-85

Gardin, S., S. M. J. van Laere, F. M. B. van Coillie, F. Anseel, W. Duyck, R. R. de Wulf \& L. P. C. Verbeke (2011) Remote sensing meets psychology: a concept for operator performance assessment. Remote Sensing Letters, 2, 251-257.

Hay, G. \& G. Castilla. (2006) Object-based image analysis: strengths, weaknesses, opportunities and threats (SWOT). In Proc. 1st Int. Conf. OBIA, 4-5.

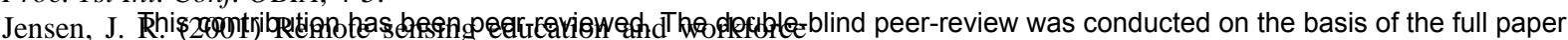
considerations. Geocarto International, 16, 63-68. doi:10.5194/isprsannals-III-6-3-2016
Lawhead, P. \& J. Johnson. (2003) Institute for Advanced Education in Geospatial Sciences Educating the Next Generation of Scientists. In NASA Conference Publication, 133-160. NASA 1998.

Lillesand, T. M., R. W. Kiefer \& J. W. Chipman. (2004) Remote sensing and image interpretation. New York, NY: John Wiley \& Sons Ltd.

Navulur, K. (2006a) Creating Objects. In Multispectral image analysis using the object-oriented paradigm. CRC press.

---. (2006b) Object-Based Image Analysis. In Multispectral image analysis using the object-oriented paradigm. CRC press.

Paine, D. P. (1981) Aerial Photography and Image Interpretation. New York, NY: Wiley.

Reinhardt, W. (2013) Statement of usage of BoK in our institute. AGILE, Munich, Germany.

Sader, S. A. \& T. Mueller (2012) Survey on Remote Sensing and Geospatial Technologies Training in U.S. and Canadian Colleges and Universities.

Photogrammetric Engineering and Remote Sensing, 78, 1193 1198.

Sader, S. A. \& S. Vermillion (2000) Remote sensing education: An updated survey. Journal of Forestry, 98, 31-37.

Stone, K. H. (1964) A Guide to the Interpretation and Analysis of Aerial Photos. Annals of the Association of American Geographers, 54, 318-328.

Sui, D. (2014) Opportunities and impediments for open GIS.

Transactions in GIS, 18, 1-24.

Tong Yang, X., H. Liu \& X. Gao (2015) Land cover changed object detection in remote sensing data with medium spatial resolution. International Journal of Applied Earth Observation and Geoinformation, 38, 129-137. 